\title{
Method for Calculating the Injection Effect to Create Air Circulation Inside Containers
}

\author{
Matluba A. Khadjimukhametova, Avaz M. Merganov
}

\begin{abstract}
The paper investigates the process of air circulation inside the container due to the effect of injection by an external stream. It is shown in the paper that injection can be created using the open hatch of the container, which is fixed at a certain angle. To calculate the emerging turbulent flow, a new effective mathematical model is used. The obtained numerical results of the hydrodynamic equations show that by a simple structural change in a simple container, air circulation inside the container can be significantly improved. This phenomenon gives a good perspective for the use of containers in the transportation of food.
\end{abstract}

Keywords: air circulation, container, injection, mathematical model, air flow, air injection

\section{INTRODUCTION}

Export of industrial and agricultural products is one of the main development factors for any country. Agricultural products are recommended to be transported in refrigerated containers where temperature and humidity can be controlled [1]. This leads to a rise in the cost of production, and most importantly, the rolling stock of most of the southern republics of the CIS does not have refrigerated containers. The purchase of refrigerated containers requires a large foreign exchange investment. Therefore, agricultural products of these republics are mainly delivered either in motor vans or by rail in refrigerated sections. However, due to the high price policy of transporting agricultural products in refrigerated sections, the transport of such goods in ordinary boxcars has often been carried out recently. However, due to the length of delivery time and the lack of conditions in conventional covered wagons for storing perishable products, rail transportation is a rather risky event. These factors severely limit the export opportunities of agricultural producers.

More relevant for these purposes is the use of conventional 20 ton containers, since any agricultural product loses its quality when reloaded from one mode of transport to another. And the use of containers makes it possible to deliver products from the field to the consumer, which greatly improves the quality of the product by reducing spoilage during transportation. However, existing containers do not provide complete air circulation inside the container. This leads to the fact that on sunny days such containers quickly heat up, which leads to spoilage of products. For this purpose, containers with a device for forced ventilation can be used. However, equipping simple

\footnotetext{
Revised Manuscript Received on 14, October 2019.

Matluba A. Khadjimukhametova, Head of Department, Tashkent Railway Engineering Institute, Tashkent, Uzbekistan(E-mail: matluba_78@mail.ru)

Avaz M. Merganov, Head of Research, Innovation and Training of

Scientific and Pedagogical Staff Tashkent Railway Engineering Institute,Tashkent, Uzbekistan(E-mail: meravaz@gmail.com)
}

containers with such fans also requires a large capital investment and time. To this problem is added the problem of the delivery of electrical energy to the fans.

In [2], methods for modifying simple containers for transporting food products are proposed. Firstly, the lining of containers from the inside is offered with affordable and inexpensive heat-shielding material. Secondly, the idea is proposed to use a stream of air flowing around the container to create circulation inside the container. For this purpose, hatches are proposed that are cut with a strip of width on the top and bottom of the container. These hatches should open at a certain angle, as shown in fig. 1. The idea of such a simple device is that when the container moves, these airways flow around these hatches. With such a flow, rarefaction of air is formed at the outlet of the upper hatch, and overpressure is formed at the entrance to the lower hatch. Consequently, an air stream enters the container through the lower hatch and exits through the upper.

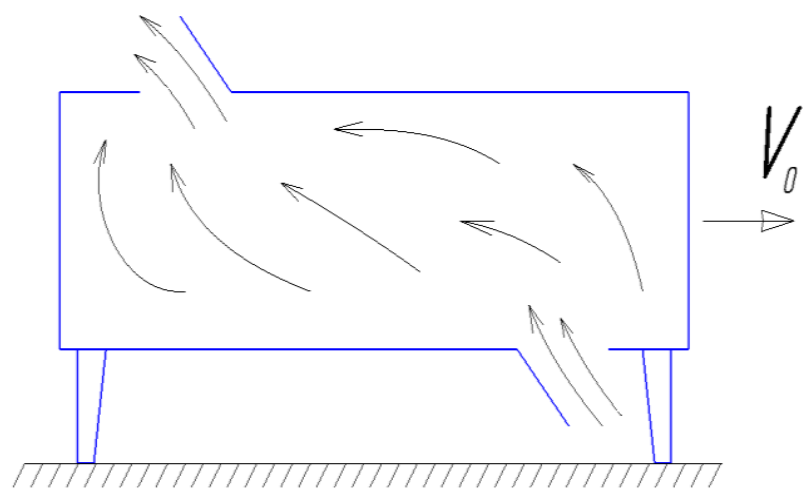

Fig. 1. Picture of air circulation inside the container.

In the figure, the direction of flow is indicated by arrows. Thus, the proposed device plays the role of a fan, which contributes to the circulation of air throughout the volume of the container. Air circulation helps lower the temperature inside the container. This is especially necessary as mentioned above on sunny days, when due to solar radiation the temperature inside the container can increase significantly. However, to achieve the described effect of air circulation inside the container, it is necessary to study the aerodynamics of the air flow around the hatch. In [2], to study the injection process in the upper hatch, a oneparameter Prandtl turbulence model was used [3]. This model gives good results, for example, for the main section of a free flooded jet, where the solution has a self-similar character. However, for the task when using this model, we can get only a qualitative picture. To obtain more accurate calculation results, it is necessary to use modern turbulence

Published By:

Blue Eyes Intelligence Engineering

\& Sciences Publication

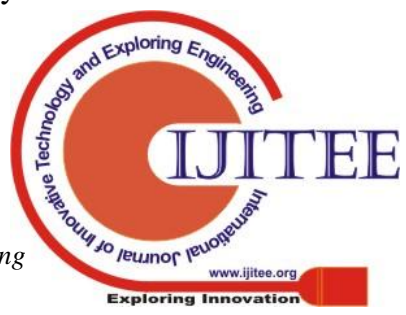




\section{METHOD FOR CALCULATING THE INJECTION EFFECT TO CREATE AIR CIRCULATION INSIDE CONTAINERS}

models. These methods are Direct Numerical Simulation (DNS) and Large Eddy Simulation (LES). However, these methods are very laborious, require high-speed supercomputers, and their wide practical use for solving complex aerodynamic problems can only begin at the end of this century [4]. Therefore, in the near future, semiempirical methods will remain the main working tool for solving applied aerodynamics problems. All semiempirical turbulence models are based on the Reynolds equation [5]. However, the so-called Reynolds voltages remain unknown in this equation. Therefore, the resulting system is not closed. To close the resulting system of equations, a large number of different mathematical models have been proposed. These models are based on the hypotheses of Boussinesq, Prandtl, Karman, Kolmogorov, etc. Having analyzed the proposed semi-empirical models, it can be argued that the models of Spalart and Allmaras [6] and the Menter model [7], [8, 9] are modern and widely used. Using these models, numerical solutions are obtained for a wide range of aerodynamic problems. Nevertheless, the closure problem for the Reynolds equations cannot be considered complete. Because in many cases it is necessary to introduce additional assumptions that are only approximate in nature. This is especially true when calculating swirling turbulent flows or flows when flowing around bodies with large curvature. This is explained by the fact that almost all existing turbulence models are based on transport theory, where factors of inertia forces are not taken into account. Therefore, when studying the flow around the hatch, one can expect that the results obtained by these existing models will inadequately describe the real flow [10]. Therefore, the flow around the edge of the hatch refers to flows with a large curvature.

\section{RESULTS \& DISCUSSIONS}

Recently, work [8] appeared, where a new mathematical model of turbulence is given. This model is based not on the closure of the Reynolds equation, but on the dynamics of two liquids. Those. the idea of the work is that a turbulent flow is represented as the movement of two fluids with different speeds, the so-called moles. Since this model is built on the basis of dynamics, it describes well the rotating flows. Therefore, this turbulence model is used to study the task.

In this work, a system of equations is obtained

$$
\left[\begin{array}{c}
\frac{\partial U_{i}}{\partial x_{i}}=0, \\
\frac{\partial \rho U_{i}}{\partial t}+\frac{\partial\left(\rho U_{j} U_{i}+\delta_{i j} p\right)}{\partial x_{j}}=\frac{\partial}{\partial x_{j}}\left(\Pi_{j i}-\rho v_{m j} v_{m i}\right), \\
\frac{\partial v_{m i}}{\partial t}+U_{j} \frac{\partial v_{m i}}{\partial x_{j}}=-v_{m j} \frac{\partial U_{i}}{\partial x_{j}}+\frac{\partial \pi_{j i}^{r}}{\partial x_{j}}+C_{s}\left|\operatorname{rot} U \times v_{m}\right|_{i}-C_{\mu} v_{m i}
\end{array}\right.
$$

In this system

$\Pi_{j i}=\rho v\left(\frac{\partial U_{i}}{\partial x_{j}}+\frac{\partial U_{j}}{\partial x_{i}}\right), \pi_{j i}^{\prime}=v_{e f}\left(\frac{\partial v_{m i}}{\partial x_{j}}+\frac{\partial v_{m j}}{\partial x_{i}}\right)$,

$v_{m i}$ - moth speed, $U_{i}$ - averaged flow rate , $\rho$ - medium density , $v$ - molecular density,

$v_{e f}$ - effective mole viscosity,
The task consists of two parts: the first is the flow around the hatch by an external stream, the second is the flow of the stream inside the container. The polar coordinate system is convenient for describing the first task. In this coordinate system, the system of equations (1) will have the form:

$$
\begin{gathered}
\frac{\partial V_{r}}{\partial t}+V_{r} \frac{\partial V_{r}}{\partial r}+\frac{V_{\varphi}}{r} \frac{\partial V_{r}}{\partial \varphi}-\frac{V_{\varphi}^{2}}{r}+\frac{1}{\rho} \frac{\partial p}{\partial r}=-\frac{\partial v_{\varphi} v_{r}}{r \partial \varphi} \\
\frac{\partial V_{\varphi}}{\partial t}+V_{r} \frac{\partial V_{\varphi}}{\partial r}+\frac{V_{\varphi}}{r} \frac{\partial V_{\varphi}}{\partial \varphi}+\frac{V_{r} V_{\varphi}}{r}+\frac{1}{r \rho} \frac{\partial p}{\partial \varphi}=-\frac{\partial v_{\varphi} v_{\varphi}}{r \partial \varphi} \\
\frac{\partial v_{r}}{\partial t}+V_{r} \frac{\partial v_{r}}{\partial r}+\frac{V_{\varphi}}{r} \frac{\partial v_{r}}{\partial \varphi}+v_{r} \frac{\partial V_{r}}{\partial r}+\frac{v_{\varphi}}{r} \frac{\partial V_{r}}{\partial \varphi}-\frac{2 v_{\varphi} V_{\varphi}}{r}= \\
=-C_{s} \frac{v_{\varphi}}{r}\left[\frac{\partial r V_{\varphi}}{\partial r}-\frac{\partial V_{r}}{\partial \varphi}\right]+\frac{1}{r^{2}} \frac{\partial}{\partial \varphi}\left(v_{e f} \frac{\partial v_{r}}{\partial \varphi}\right)-C_{\mu} v_{r} \\
\frac{\partial v_{\varphi}}{\partial t}+V_{r} \frac{\partial v_{\varphi}}{\partial r}+\frac{V_{\varphi}}{r} \frac{\partial v_{\varphi}}{\partial \varphi}+v_{r} \frac{\partial V_{\varphi}}{\partial r}+\frac{v_{\varphi}}{r} \frac{\partial V_{\varphi}}{\partial \varphi}+\frac{v_{\varphi} V_{r}}{r}+\frac{v_{r} V_{\varphi}}{r}= \\
=C_{s} \frac{v_{r}}{r}\left[\frac{\partial r V_{\varphi}}{\partial r}-\frac{\partial V_{r}}{\partial \varphi}\right]+\frac{1}{r^{2}} \frac{\partial}{\partial \varphi}\left(v_{e f} \frac{\partial v_{\varphi}}{\partial \varphi}\right)-C_{\mu} v_{\varphi}
\end{gathered}
$$

In this system $V_{r}, V_{\varphi}, \rho, p$ - accordingly, radial velocity, azimuthal velocity, density and pressure of the medium. In system (2), in order to facilitate the numerical calculation, the terms containing derivatives with respect to $r$ can be neglected due to smallness. Molecular viscosity is also neglected. The drag coefficient was taken equal

$$
=0.3 \sqrt{2\left[\frac{\partial V_{r}}{\partial r}\right]^{2}+2\left[\frac{1}{r} \frac{\partial V_{\varphi}}{\partial \varphi}+\frac{V_{r}}{r}\right]^{2}+\left[\frac{\partial V_{\varphi}}{\partial r}-\frac{V_{\varphi}}{r}+\frac{1}{r} \frac{\partial V_{r}}{\partial \varphi}\right]^{2}}
$$

The effective viscosity of moles was taken equal

$$
v_{e f}=0.45 \frac{v_{r} v_{f}}{C_{\mu}}
$$

The coefficient for shear force was equal $C_{s}=0.2$.

As for the second task - the flow of the stream inside the container, the air passes between containers with products (fruits). Therefore, for mathematical modeling, we adopt the Darcy law: $\vec{V}=-\boldsymbol{k}$ gradp

Substitution of this expression into the continuity equation will give us the desired equation:

$$
\operatorname{div} \boldsymbol{k g r a d} \boldsymbol{p}=0
$$

If we consider the coefficient as a constant value, we get a fairly simple Laplace equation:

$\Delta \boldsymbol{p}=0$

Thus, joining two tasks, we can solve the problem.

For a numerical study, the system of equations of hydrodynamics is carried out to a dimensionless form. To do this, all speeds are related to the speed of the container - $V_{0}$, the dimensions are related to the width of the hatch, and the pressure is related to the value $-\rho V_{0}^{2}$. The dimensionless coefficient is taken equal to $\mathrm{k}=0.05$. For the numerical implementation of system of equations (2), the explicit finite difference MacCormack scheme [10] was used. The stationary solution was achieved by the establishment method. For equation (3), the method of iterations of the upper relaxation [10] was used, which was used at each time step of the McCormack scheme. Figure 2 illustrates the result of a numerical solution of the problem for the hatch angle $60^{\circ}$. 


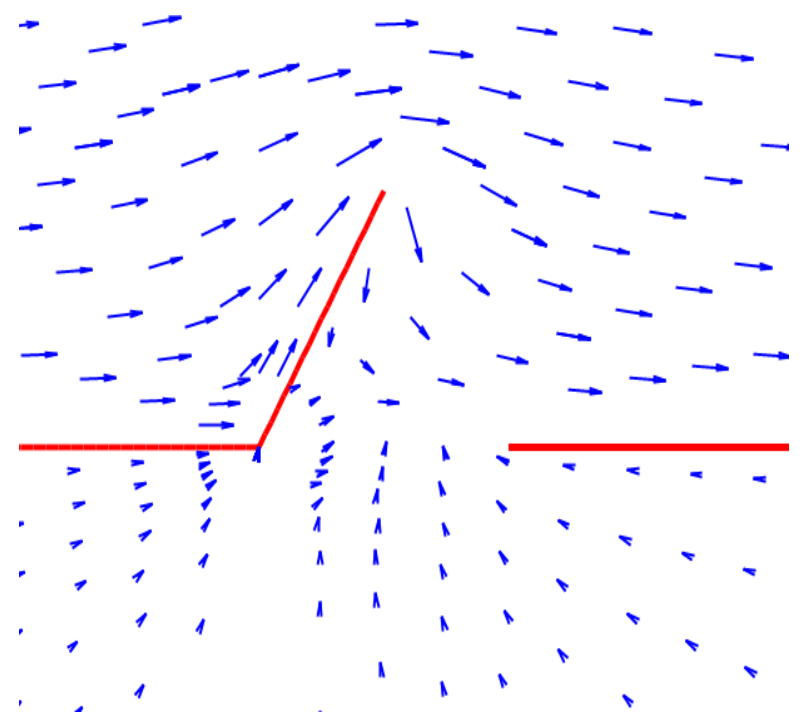

Fig. 2. A picture of the flow of air around an open hatch.

This figure shows a picture of the flow of air flow during movement of the container. It can be seen from the figure that the open hatch, through injection, draws in air from the container. The opposite picture occurs in the lower hatch. Here, on the contrary, external air is forced into the container. Thus, external air enters through the lower hatch and exits through the upper. This process can significantly prevent food from overheating.

To study the most efficient operation of the hatch numerically, we calculated the rarefaction of the flow at its various angles. Figure 3 shows a graph of the dimensionless value of the vacuum pressure on the values of the angle of the hatch. It can be seen from this graph that the greatest flow injection effect is obtained if the hatch is installed at an angle of about $30^{\circ}$.

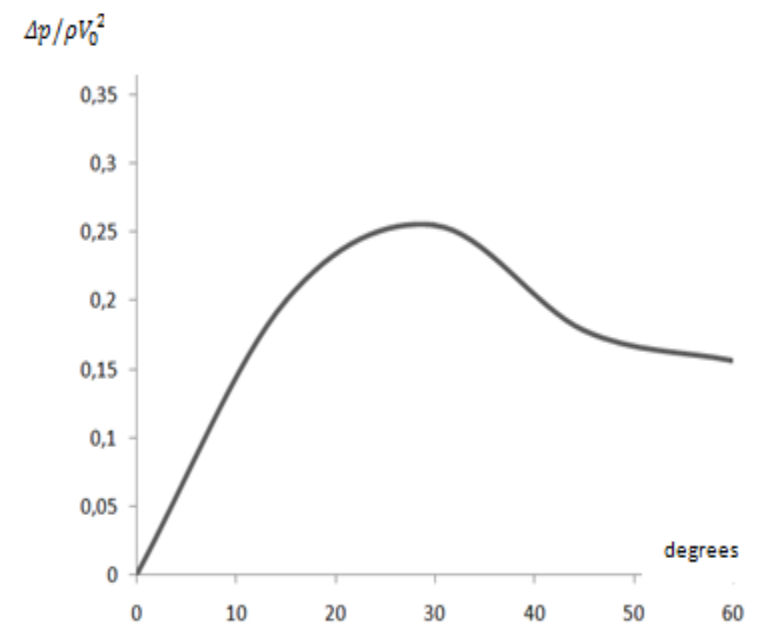

Fig. 3. Change in vacuum pressure depending on the angle of the hatch.

\section{CONCLUSION.}

Thus, the work shows that as a result of small structural solutions, it is possible to create air circulation inside the container. This decision will allow the transportation of perishable products in modified containers and will increase the export potential of the republic.

\section{LITERATURE}

1. Dzyuba I.S. transportation of perishable goods./P.1., Gomel 1999.

2. Malikov Z.M., Khadjimukhmaetova M.A. Methods for modifying simple containers for the transport of perishable goods . №1, 2017, page.58.

3. Abramovich G.N. Theory of turbulent jets/Moscow «Science», 1984

4. Spalart P. R., Allmaras S. R. "A one-equation turbulence model for aerodynamic flows"// AIAA Paper 1992-0439.

5. Menter F. R. "Zonal two-equation k- $\omega$ turbulence models for aerodynamic flows"// AIAAPaper 1993- 2906.

6. Menter F. R., Kuntz M., and Langtry R. "TenYears of Industrial Experience with the SST Turbulence Model"// Turbulence, Heat and Mass Transfer 4, ed: K. Hanjalic, Y. Nagano, and M. Tummers, Begell House, Inc., 2003, pp. $625-632$.

7. Anderson D., Tannehill J., Pletcher R. Computational fluid mechanics and heat transfer. -Moscow: Mir, 1990, Vol.1

8. Patankar $S$. Numerical heat transfer and fluid flow./Hemiphere Publishing Corporation, New York, 1980.

9. Merganov A. M. Model of search for the placement of warehouses of tarnet-and-string cargo depending on the cargo //Journal of TIRE. - 2018. - T. 14. - №. 2. - C. 129-133.

10. Avaz M. Merganov, Daurenbek I. Ilesaliev, Alexander A. Svetashev, Method of accommodation of tarned-piece cargoes in auto-vehicle // Innotrans. 2019, \#3 (33), 32-37 pp. DOI:10.20291/2311-164X-2019-3-32-36 